\section{The sequencing of chemotherapy and radiotherapy in breast cancer patients after mastectomy}

\author{
Hak Jae Kim¹, Jae-Sung Kim¹, Eui Kyu Chie', Dong-young Noh², \\ Yung-jue Bang ${ }^{3}$, Sung Whan $\mathrm{Ha}^{1,4}$ \\ Department of ${ }^{1}$ Radiation Oncology, ${ }^{2}$ Surgery, and ${ }^{3}$ Internal Medicine, Seoul National University \\ College of Medicine; ${ }^{4}$ Institute of Radiation Medicine, Seoul National University, Seoul, Korea
}

\section{ABSTRACT}

Background. The purpose of the study was to retrospectively evaluate the outcome according to the sequencing of radiotherapy and chemotherapy after mastectomy in high-risk patients with breast cancer.

Methods. From January 1986 through September 2000, 275 women with stage I-IIIB breast cancer were treated with chemotherapy and radiotherapy after mastectomy. The patients were divided into four groups. Chemotherapy was given first in 116 patients (CTRT), concurrent chemoradiotherapy in 77 (CCRT), sandwich therapy in 65 (SAND), and radiotherapy first in 17 (RTCT). Prognostic factors such as age, primary tumor size and nodal status were not statistically different among the four groups. There was a higher proportion of patients with close or positive margins in CCRT and RTCT groups than in the CTRT and SAND groups $(22 / 77,5 / 17$ vs $3 / 116,2 / 65, P$ $<0.001)$.

Results. Median follow-up was 145 months (range, 10-210). Five-year overall and disease-free survival were $69.4 \%$ and $56.1 \%$, respectively. Survival outcomes were not statistically different among the four groups (5-year overall/disease-free survival, 68.0\%/63.0\%, 71.3\%/60.8\%, 65.0\%/48.1\%, 81.9\%/58.8\%, in CTRT, CCRT, SAND, and RTCT, respectively) $(P=0.3422 / P=0.6333)$. The incidence of local-regional recurrence was not different in the early radiotherapy group (CCRT/RTCT, 11\%/12\%) and delayed radiotherapy group (CTRT/SAND, 7\%/8\%).

Conclusions. This study suggests that in these high-risk breast cancer patients after mastectomy, delay in the start of radiotherapy does not increase local-regional recurrence, and the final survival outcomes are not affected by the sequencing of chemotherapy and radiotherapy. Free full text available at www.tumorionline.it

\section{Introduction}

There has been a renewed interest in postmastectomy radiotherapy (PMRT) in breast cancer since the publication of two modern trials that demonstrated a survival advantage with the addition of radiotherapy and systemic chemotherapy in node-positive breast cancer patients after mastectomy ${ }^{1,2}$. Based on these studies, the American Society of Clinical Oncology in 2001 recommended PMRT in patients with four or more positive axillary lymph nodes, with T3 or stage III tumors ${ }^{3}$. Historically, most institutions have administered chemotherapy followed by radiotherapy in postmastectomy patients. This conventional sequence is based on the ability of chemotherapy to reduce metastatic spread in women with positive and negative nodes, with the consideration of less of an effect of local-regional control after PMRT on overall survival. However, this argument may not be valid, given the recent findings of PMRT trials ${ }^{1,2}$.

The optimal sequence of radiotherapy with chemotherapy after mastectomy is still considered an unanswered question ${ }^{4}$. Previous retrospective studies that have ana-

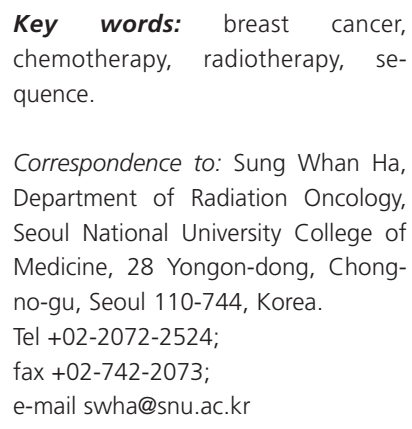

Correspondence to: Sung Whan $\mathrm{Ha}$, Department of Radiation Oncology, Seoul National University College of Medicine, 28 Yongon-dong, Chongno-gu, Seoul 110-744, Korea. Tel +02-2072-2524;

fax +02-742-2073;

e-mail swha@snu.ac.kr

Received December 23, 2008; accepted June 12, 2009. 
lyzed the consequences of delaying the initiation of radiotherapy to deliver a full course of chemotherapy after mastectomy have been controversial ${ }^{5-7}$. Despite this unsolved question, a recent Patterns of Care survey demonstrated that chemotherapy was delivered and was completed before PMRT in $95 \%$ of the cases $^{8}$. The question of whether a conventional sequence of a full course of chemotherapy that was first followed by radiotherapy is optimal is intriguing. A trial from Denmark (radiotherapy administered after the first cycle of chemotherapy) and a trial from British Columbia, Canada (radiotherapy administered after the fourth cycle of chemotherapy), showed a significant survival benefit in favor of radiotherapy delivered between successive cycles of chemotherapy rather than after the completion of chemotherapy ${ }^{1,2}$.

The purpose of the present study was to evaluate retrospectively the importance of the sequence of radiotherapy and chemotherapy on outcomes such as overall survival (OS), disease-free survival (DFS) and failure patterns after mastectomy in high-risk patients with breast cancer.

\section{Materials and methods}

\section{Patient characteristics}

Between January 1986 and September 2000, 275 women with stage I-IIIB breast cancer were treated with adjuvant chemotherapy (with or without tamoxifen) and radiotherapy after mastectomy. The median age of patients at the time of diagnosis was 42 years (range, 21$71)$. At the time of diagnosis, 215 patients $(79 \%)$ were 50 years of age or younger. To analyze retrospectively whether the sequence of postoperative adjuvant chemotherapy and radiotherapy after mastectomy influenced the results of treatment, the population in the study was divided into four groups. Chemotherapy was given first in 116 patients (the CTRT group). Radiotherapy was given first in 17 patients (the RTCT group). Sandwich therapy was given for 65 patients (the SAND group), where two or three chemotherapy cycles were given first followed by radiotherapy (without concurrent chemotherapy) and then the remaining chemotherapy cycles were administered. In 77 patients, concurrent chemoradiotherapy was given to 77 patients (the CCRT group). The sequence of adjuvant treatments was decided in consultation by the medical and radiation oncologists. The clinical and pathological characteristics of the patients are described in Table 1.

\section{Treatment}

Surgery. In most patients (256/275, 93\%), a modified radical mastectomy with axillary lymph node dissection was performed. Sixteen patients $(6 \%)$ underwent a radical mastectomy and 3 patients (1\%) underwent a sim-

\begin{tabular}{lc}
$\begin{array}{l}\text { Table } \mathbf{1} \text { - Clinical and pathological characteristics of } \mathbf{2 7 5} \\
\text { patients }\end{array}$ \\
\hline Characteristics & No. of patients (\%) \\
\hline Age (yr), median & 42 \\
$<50$ & $215(78)$ \\
$\geq 50$ & $60(22)$ \\
ECOG performance status & $9(3)$ \\
0 & $262(95)$ \\
1 & $4(2)$ \\
2 & $3(1)$ \\
Surgery & $256(93)$ \\
Simple mastectomy & $16(6)$ \\
Modified radical mastectomy & \\
Radical mastectomy & $77(28)$ \\
Chemo-RT sequencing & $116(42)$ \\
CCRT & $17(6)$ \\
CTRT & $65(24)$ \\
RTCT &
\end{tabular}

ECOG, Eastern Cooperative Oncology Group; CCRT, concurrent chemoradiotherapy; CTRT, chemotherapy-radiotherapy; RTCT, radiotherapy-chemotherapy; SAND, sandwich.

ple mastectomy. Twenty-one patients had positive resection margins and 11 patients had close $(\leq 2 \mathrm{~mm})$ resection margins as determined on pathological examination. A median of 18 axillary lymph nodes (range, 158) were removed during surgery.

Radiotherapy. Indications for undergoing postoperative radiotherapy were one or more of the following: a tumor size $\geq 5 \mathrm{~cm}$, four or more positive lymph nodes, a $\mathrm{T} 4$ primary cancer and close $(\leq 2 \mathrm{~mm})$ or positive resection margins. All of the patients received radiotherapy with the use of photons from a ${ }^{60} \mathrm{Co}$ unit (100/275) until October 1992, or with megavoltage radiation (175/275), electrons or a combination of the two modalities. A median dose of 50.4 Gy (49.5-60.4), given in 28 fractions over a period of 5.5 weeks, was administered to the chest wall, axillary, supraclavicular, and internal mammary lymph node area. For linear accelerator-based treatments, radiation was usually given with electron beam treatments to the chest wall combined with photons to the axilla and supraclavicular areas, along with irradiation of the internal mammary lymph node area with photons for the first 14 fractions followed by electrons for the remaining 14 fractions.

Chemotherapy. None of the patients analyzed underwent chemotherapy or hormonal therapy prior to mastectomy. Chemotherapy regimens used during the study period were CMF (cyclophosphamide, methotrxate, 5-fluorouracil), CMF-prednisolone, CAMF (CMF plus doxorubicin), CAF (cyclophosphamide, doxorubicin, 5-fluorouracil), AC (doxorubicin plus cyclophosphamide), CEF (cyclophosphamide, epirubicin, 5-fluorouracil), $\mathrm{AC}+\mathrm{T}$ (four cycles of doxorubicin plus cyclophosphamide followed by four cycles of paclitaxel), 
and CAF + T ( 6 cycles of CAF followed by four cycles of paclitaxel). CMF was the most commonly used regimen (150/275, 55\%). The median number of chemotherapy cycles administered was six (range, 5-12).

\section{Statistical analysis}

OS was defined as the length of the time until death regardless of cause, and DFS was defined as the length of time before the first evidence of recurrence including local-regional recurrence (LRR) or the presence of a distant metastasis. Survival analysis was performed using the Kaplan-Meier method. The chi-squared test was used to determine the difference of prognostic factors among the four patient groups. Differences of survival, local-regional control and patterns of failure among the four patient groups were assessed by use of the logrank test, Fisher's exact test and Pearson's chi-squared test. All $P$ values were two-sided, and a value of $P \leq 0.05$ was considered statistically significant. Statistical analysis was performed by use of SPSS software (release 12.0.1, SPSS, Chicago, IL, USA).

\section{Results}

The median follow-up period was 145 months (range, 10-210). The patient characteristics are listed in Tables 1 and 2. The median tumor size was $4.5 \mathrm{~cm}$ (range, 0.5-

Table 2 - Patient characteristics according to the four patient groups

\begin{tabular}{lccccc}
\hline & $\begin{array}{c}\text { CCRT } \\
(n=77) \\
\text { No. (\%) }\end{array}$ & $\begin{array}{c}\text { CTRT } \\
(n=116) \\
\text { No. }(\%)\end{array}$ & $\begin{array}{c}\text { RTCT } \\
(n=17) \\
\text { No. }(\%)\end{array}$ & $\begin{array}{c}\text { SAND } \\
(n=65) \\
\text { No. }(\%)\end{array}$ & $P$ \\
\hline Age & & & & & \\
$<50$ & $62(81)$ & $90(78)$ & $16(94)$ & $47(72)$ & 0.347 \\
$\geq 50$ & $15(19)$ & $26(22)$ & $1(6)$ & $18(28)$ & \\
ER status & & & & & \\
$\quad$ Positive & $9(12)$ & $35(30)$ & $2(11)$ & $19(29)$ & \\
Negative & $20(26)$ & $47(41)$ & $4(24)$ & $26(40)$ & 0.060 \\
$\quad$ Unknown & $48(62)$ & $34(29)$ & $11(65)$ & $20(31)$ & \\
Margin & & & & & \\
Positive & $14(18)$ & $1(1)$ & $5(29)$ & $1(2)$ & \\
Close & $8(11)$ & $2(2)$ & $0(0)$ & $1(2)$ & $<0.001$ \\
Negative & $55(71)$ & $113(97)$ & $12(71)$ & $63(96)$ & \\
T stage & & & & & \\
T1 & $7(9)$ & $19(16)$ & $3(18)$ & $3(5)$ & \\
T2 & $35(45)$ & $67(58)$ & $9(53)$ & $32(49)$ & 0.573 \\
T3 & $33(43)$ & $27(23)$ & $5(29)$ & $27(41)$ & \\
T4 & $2(3)$ & $3(3)$ & $0(0)$ & $3(5)$ & \\
N stage & & & & & \\
N0 & $11(14)$ & $9(8)$ & $5(29)$ & $0(0)$ & \\
N1 & $12(16)$ & $16(14)$ & $3(18)$ & $13(20)$ & 0.347 \\
N2 & $24(31)$ & $44(28)$ & $3(18)$ & $15(23)$ & \\
N3 & $30(39)$ & $47(40)$ & $6(35)$ & $37(57)$ & \\
\hline
\end{tabular}

ER, estrogen receptor; CCRT, concurrent chemoradiotherapy; CTRT, chemotherapy-radiotherapy; RTCT, radiotherapy-chemotherapy; SAND, sandwich.
13), and infiltrating ductal carcinoma was the most common histological subtype (262/275, 95\%). Sixty-five (24\%) of 275 patients were estrogen-receptor positive and 97 patients $(35 \%)$ were estrogen-receptor negative. The median number of involved lymph nodes was 8 (range, 0-46). Prognostic factors, including age, T stage, $\mathrm{N}$ stage and estrogen receptor status were no different among the four patient groups, but a higher proportion of patients in the CCRT and RTCT groups had positive or close resection margins (Table 2).

Five-year OS and DFS rates for all the patients were $69.4 \%$ and $56.1 \%$, respectively (Figure 1 ). According to patient group, 5-year OS and DFS were $71.3 \%$ and $60.8 \%$ for the CCRT group, $68.0 \%$ and $63.0 \%$ for the CTRT group, $81.0 \%$ and $58.8 \%$ for the RTCT group, and $65.0 \%$ and $48.1 \%$ for the SAND group, respectively. There were no significant differences among the four patient groups with respect to OS $(P=0.36$, Table 3$)$ and DFS ( $P$ $=0.63$, Table 3 ).

Among the 275 patients, there were 114 treatment failures identified during the follow-up period. Patterns of failure of the 114 patients were as follows. The presence of a distant metastasis only in 90 patients, chest wall failure only in 10 patients, regional nodal failure (supraclavicular, axillary and internal mammary lymph node

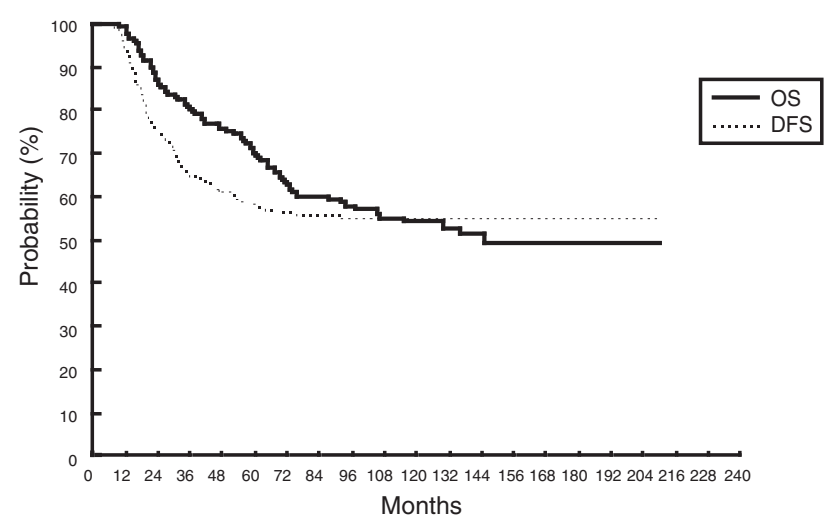

Figure 1 - Overall and disease-free survival of the 275 patients.

Table 3 - Five- and 10-year overall (OS) and disease-free survival (DFS) rates in the four patient groups

\begin{tabular}{llllll}
\hline & CCRT & CTRT & RTCT & SAND & $P$ \\
\hline OS & & & & & \\
$\quad 5-\mathrm{yr}$ & 71.3 & 68.0 & 81.9 & 65.0 & 0.3422 \\
$\quad$ 10-yr & 61.0 & 50.0 & 68.0 & 43.0 & \\
DFS & & & & & \\
5-yr & 60.8 & 63.0 & 58.8 & 48.1 & 0.6333 \\
10-yr & 55.9 & 53.9 & 58.8 & 48.1 & \\
\hline
\end{tabular}

CCRT, concurrent chemoradiotherapy; CTRT, chemotherapy-radiotherapy; RTCT, radiotherapy-chemotherapy; SAND, sandwich. 
area) only in 5 patients, chest wall failure and the presence of a distant metastasis in 5 patients, and regional node failure and the presence of a distant metastasis in 4 patients.

There were no significant differences in the rates of LRR only, LRR and the presence of a distant metastasis, or the presence of a distant metastasis only among the four patient groups ( $P=0.51$, Table 4$)$. Most patients with treatment failures had systemic recurrences (28$40 \%$ ). The LRR rate was approximately $4-7 \%$, and the LRR with concomitant systemic failure rate was $3-6 \%$ in the four patient groups.

To analyze the influence of the time interval from the date of surgery to the date of radiotherapy administration on the LRR, the patients were subdivided into three categories ( $\leq 2$ months, $>2$ months $\sim \leq 6$ months, $>6$ months) according to the surgery-radiotherapy interval (SRI). The SRI did not influence the LRR rate. The incidence of LRR was $11.7 \%$ for the CCRT and RTCT groups (SRI $\leq 2$ months), $7.6 \%$ in the CTRT group (SRI $>6$ months) and $6.8 \%$ in the SAND group (SRI 2 months $<\leq 6$ months), with no statistical significance $(P=0.48$, Table 5$)$.

In a separate analysis of a subgroup of 32 patients with positive or close resection margins, the 5 -year OS and DFS were $84.0 \%$ and $65.0 \%$, respectively. In these patients, 11 treatment failures occurred with systemic failure alone in 10 patients and LRR alone (with chest wall failure) in 1 patient. For OS, there was a trend toward better survival in patients who underwent early radiotherapy (patients in the RTCT or CCRT group) than for patients where radiotherapy was delayed (patients in the CTRT or SAND group) $(P=0.0359$, Figure 2$)$.

Table 4 - Patterns of failure in the four patient groups

\begin{tabular}{lccccc}
\hline & $\begin{array}{c}\text { CCRT } \\
(n=77) \\
\text { No. }(\%)\end{array}$ & $\begin{array}{c}\text { CTRT } \\
(n=116) \\
\text { No. }(\%)\end{array}$ & $\begin{array}{c}\text { RTCT } \\
(n=17) \\
\text { No. }(\%)\end{array}$ & $\begin{array}{c}\text { SAND } \\
(n=65) \\
\text { No. }(\%)\end{array}$ & $P$ \\
\hline LRR & $6(7)$ & $5(4)$ & $1(6)$ & $3(5)$ & \\
LRR + DM & $3(4)$ & $3(3)$ & $1(6)$ & $2(3)$ & 0.507 \\
DM only & $24(31)$ & $33(28)$ & $5(29)$ & $28(40)$ & \\
Total & $33(43)$ & $41(35)$ & $7(41)$ & $33(50)$ & \\
\hline
\end{tabular}

LRR, local-regional relapse; DM, distant metastasis; CCRT, concurrent chemoradiotherapy; CTRT, chemotherapy-radiotherapy; RTCT, radiotherapy-chemotherapy; SAND, sandwich.

Table 5 - Local-regional recurrence (LRR) rates according to the surgery-radiotherapy interval (SRI)

\begin{tabular}{lccc}
\hline Group & SRI (mo) & LRR (\%) & $P$ \\
\hline CCRT, RTCT & $<2$ & $11 / 94(11)$ & \\
SAND & $\geq 2 \sim<6$ & $5 / 65(8)$ & 0.480 \\
CTRT & $\geq 6$ & $8 / 116(7)$ & \\
\hline
\end{tabular}

CCRT, concurrent chemoradiotherapy; CTRT, chemotherapy-radiotherapy; RTCT, radiotherapy-chemotherapy; SAND, sandwich.

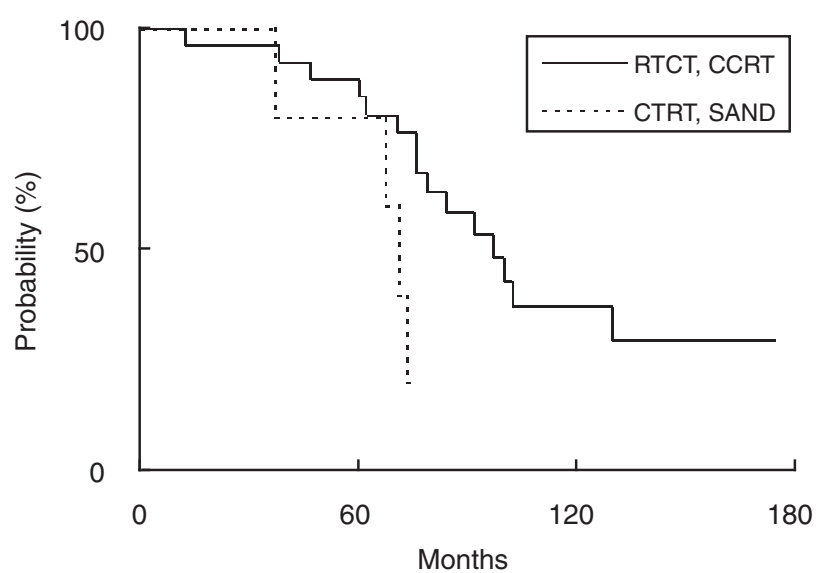

Figure 2 - Overall survival curves showing that patients who received early radiotherapy (in the RTCT and CCRT groups) had significantly better outcomes than patients where radiotherapy was delayed (in the CTRT and SAND groups) for patients with a close or positive resection tumor margin $(P=0.0359)$.

\section{Discussion}

PMRT substantially reduces the risk of LRR for highrisk women with breast cancer. However, the impact of such a reduction on the risk of distant failure and ultimately death due to cancer has been controversial. The publication of long-term results of the two largest trials conducted for evaluation of the use of PMRT for premenopausal node-positive patients treated with chemotherapy has brought this debate renewed attention $^{1,2}$. These studies showed that the administration of radiotherapy following a modified radical mastectomy not only reduced local-regional failure rates but also yielded clinically relevant improvements in DFS and OS rates in premenopausal patients who received chemotherapy.

Considering the impact according to the administration sequence of radiotherapy and chemotherapy in these high-risk patients, early administration of systemic chemotherapy can theoretically reduce systemic relapse, whereas early radiotherapy can reduce LRR. In this regard, optimal integration of chemotherapy and radiotherapy as adjuvant treatments in patients initially treated with a mastectomy is important to maximize the therapeutic outcome.

Most studies evaluating the consequence by sequence of chemotherapy and radiotherapy have been performed with the use of breast-conserving therapy of early breast cancer ${ }^{9-14}$. However, there are few data suggesting the optimal sequencing in patients after mastectomy. Recently, Piroth et al..$^{15}$ reported a retrospective study of 212 cases treated with chemotherapy and radiotherapy after mastectomy. The patients were divided into 3 groups according to the sequence of chemotherapy and radiotherapy (SEQ-group: radiotherapy was ap- 
plied after finishing the last chemotherapy cycle, SWgroup: 2-4 chemotherapy cycles were given prior to radiotherapy followed by 2-4 further chemotherapy cycles, SIM-group: chemotherapy and radiotherapy were applied simultaneously). There was no significant difference in treatment outcome in relation to the sequencing of chemotherapy and PMRT. These findings were similar to those of our studies. That is, survival outcomes and patterns of treatment failure were no different according to treatment sequence.

Our study also analyzed the influence of the interval from the date of surgery to the date of radiotherapy administration (SRI) on LRR. There were conflicting results on this issue. In one study, the 8-year actuarial risk of LRR among 19 patients beginning radiotherapy within 6 months of the initial diagnosis was 5\%, compared to $23 \%$ among 35 patients initiating radiotherapy more than six months after the diagnosis ${ }^{6}$. The investigators suggested that a delay in the initiation of radiotherapy for a period of six or more months from the time of diagnosis resulted in a higher local failure rate for patients requiring chemotherapy and radiotherapy for local-regional breast cancer.

Conversely, numerous studies found no relationship between SRI and local-regional failure. A retrospective study of 221 patients receiving PMRT between 1977 and 1992 showed little, if any, impact on the interval between surgery and PMRT for the risk of LRR. Adjuvant chemotherapy was given to 151 patients. With a median follow-up of 4.3 years, the LRR rates at 5 years were $13 \%$ for 82 patients who began PMRT within 2 months of a mastectomy, $4 \%$ for 50 patients who began PMRT from 2.1 to 6 months after surgery, and $9 \%$ for 89 patients who began PMRT more than 6 months after surgery $(P=$ $0.51)^{7}$. It was also ascertained in two randomized trials that delaying PMRT was not related with $\mathrm{LRR}^{16,17}$. Our study similarly showed that the SRI did not influence the LRR rate. The incidence of LRR was $11.7 \%$ for the CCRT and RTCT groups (SRI $\leq-2$ months), $7.6 \%$ in the CTRT group (SRI $>6$ months), and $6.8 \%$ in the SAND group (SRI 2 months $<\leq 6$ months), with no statistical significance.

Although the optimal sequencing of chemotherapy and PMRT cannot be determined from available evidence, current guidelines recommend that chemotherapy should be started soon after surgery, and hence the start of chemotherapy should not be delayed until after PMRT $^{3,18}$.

However, in cases with close or positive margin status, determination of treatment sequencing should be made cautiously. In the present study, there was a small number of patients with a positive or close resection margin ( $n=32)$. Of these patients, most $(27 / 32)$ received early radiotherapy (patients in the CCRT and RTCT groups). When we analyzed a subgroup of 32 patients for treatment outcomes, patients who received early radiotherapy (patients in the CCCT and RTCT groups) had a trend toward better OS than patients who received delayed radiotherapy (patients in the CTRT and SAND groups) ( $P$ $=0.0359$, Figure 2 ).

There are several limitations to the present study. First, only 275 patients were analyzed retrospectively. Furthermore, the number of patients in the RTCT group was small compared with the other treatment groups, so that the study was underpowered to detect small but potential differences in treatment outcome. Secondly, a higher proportion of patients in the CCRT and RTCT groups had positive or close resection margins. Such imbalances in characteristic distributions could suggest caution in the interpretation of treatment outcome of the study.

\section{Conclusions}

There are few data suggesting how to sequence chemotherapy and radiotherapy after mastectomy. Generally, radiotherapy should follow chemotherapy according to current guideline in such patients ${ }^{3}$. Our results confirmed these findings, indicating that for the use of PMRT, the sequence of radiotherapy and chemotherapy does not affect the final survival outcome or recurrence patterns of patients with high-risk breast cancer. However, for patients with positive or close resection margins, early integration of radiotherapy might be considered as there was a trend toward better survival in patients who received early radiotherapy (patients in the RTCT and CCRT groups) than for patients who received delayed radiotherapy (patients in the CTRT and SAND groups). LRR rates were the same for the patients in the four groups despite high rates of a close or positive margin for patients in the RTCT and CCRT groups.

\section{References}

1. Overgaard M, Hansen PS, Overgaard J, Rose C, Andersson M, Bach F, Kjaer M, Gadeberg CC, Mouridsen HT, Jensen $\mathrm{M}$, Zedeler K: Postoperative radiotherapy in high-risk premenopausal women with breast cancer who receive adjuvant chemotherapy. Danish Breast Cancer Cooperative Group 82b Trial. N Engl J Med, 337: 949-955, 1997.

2. Ragaz J, Jackson SM, Le N, Plenderleith IH, Spinelli JJ, Basco VE, Wilson KS, Knowling MA, Coppin CML, Paradis M, Coldman AJ, Olivotto IA: Adjuvant radiotherapy and chemotherapy in node-positive premenopausal women with breast cancer. N Engl J Med, 337: 956-962, 1997.

3. Recht A, Edge SB, Solin LJ, Robinson DS, Estabrook A, Fine RE, Fleming GF, Formenti S, Hudis C, Kirshner JJ, Krause DA, Kuske RR, Langer AS, Sledge GW, Jr, Whelan TJ, Pfister DG: Postmastectomy radiotherapy: clinical practice guidelines of the American Society of Clinical Oncology. J Clin Oncol, 19: 1539-1569, 2001.

4. Hebert-Croteau N, Freeman CR, Latreille J, Brisson J: Delay in adjuvant radiation treatment and outcomes of breast cancer - a review. Breast Cancer Res Treat, 74: 77-94, 2002. 
5. Buzdar AU, Kau SW, Smith TL, Ames F, Singletary E, Strom E, McNeese, M, Hortobagyi GN: The order of administration of chemotherapy and radiation and its effect on the local control of operable breast cancer. Cancer, 71: 36803684, 1993.

6. Buchholz TA, Austin-Seymour MM, Moe RE, Ellis GK, Livingston RB, Pelton JG, Griffin TW: Effect of delay in radiation in the combined modality treatment of breast cancer. Int J Radiat Oncol Biol Phys, 26: 23-35, 1993.

7. Metz JM, Schultz DJ, Fox K, Mathews A, Glick J, Solin LJ: Analysis of outcomes for high-risk breast cancer based on interval from surgery to postmastectomy radiation therapy. Cancer, 6: 324-330, 2000.

8. White J, Moughan J, Pierce LJ, Morrow M, Owen J, Wilson JF: Status of postmastectomy radiotherapy in the United States: a patterns of care study. Int J Radiat Oncol Biol Phys, 60: 77-85, 2004.

9. Recht A, Come SE, Henderson IC, Gelman RS, Silver B, Hayes DF, Shulman LN, Harris JR: The sequencing of chemotherapy and radiation therapy after conservative surgery for early-stage breast cancer. N Engl J Med, 334: 1356-1361, 1996.

10. Calais G, Serin D, Forquet A, Bosset J, Favre A: Randomized study comparing adjuvant radiotherapy (RT) with concomitant chemotherapy (CT) versus sequential treatment after conservative surgery for patients with stage I and II breast carcinoma. Int J Radiat Oncol Biol Phys, 54(Suppl 2): 57-58, 2002.

11. Recht A: Integration of systemic therapy and radiation therapy for patients with early-stage breast cancer treated with conservative surgery. Clin Breast Cancer, 4: 104-113, 2003.

12. Bellon JR, Come SE, Gelman RS, Henderson IC, Schulman
LN, Silver BJ, Harris JR, Recht A: Sequencing of chemotherapy and radiation therapy in early-stage breast cancer: updated results of a prospective randomized trial. J Clin Oncol, 23: 1934-1940, 2005.

13. Hartsell WF, Recine DC, Griem KL, Murthy AK: Delaying the initiation of intact breast irradiation for patients with lymph node positive breast cancer increases the risk of local recurrence. Cancer, 76: 2497-2503, 1995.

14. Leonard CE, Wood ME, Zhen B, Rankin J, Waitz DA, Norton L, Howell K, Sedlacek S: Does administration of chemotherapy before radiotherapy in breast cancer patients treated with conservative surgery negatively impact local control? J Clin Oncol, 13: 2906-2915, 1995.

15. Piroth MD, Pinkawa M, Gagel B, Stanzel S, Asadpour B, Eble MJ: Sequencing chemotherapy and radiotherapy in locoregional advanced breast cancer patients after mastectomy - a retrospective analysis. BMC cancer, 8: 114-122, 2008.

16. Griem KL, Henderson IC, Gelman R, Ascoli D, Silver B, Recht A, Goodman RL, Hellman S, Harris JR: The 5-year results of a randomized trial of adjuvant radiation therapy after chemotherapy in breast cancer patients treated with mastectomy. J Clin Oncol, 5: 1546-1555, 1987.

17. Olson JE, Neuberg D, Pandya KJ, Richter MP, Solin LJ, Gilchrist KW, Tormey DC, Veeder M, Falkson G: The role of radiotherapy in the management of operable locally advanced breast cancer: Results of a randomized trial by the Eastern Cooperative Oncology Group. Cancer, 79: 11381149, 1997.

18. Goldhirsch A, Glick JH, Gelber RD, Coates AS, Thurlimann B, Senn HJ: Meeting highlights: international expert consensus on the primary therapy of early breast cancer 2005 . Ann Oncol, 16: 1569-1583, 2005. 